\title{
Development of Central Serous Chorioretinopathy following Chalazion Removal with Intralesional Triamcinolone Injection: A Case Report
}

\author{
Luca Rosignoli Stephen Myles Potter Andres Gonzalez Sarina Amin \\ Syed G. Khurshid \\ Department of Ophthalmology, University of Florida College of Medicine, \\ Gainesville, FL, USA
}

\section{Keywords}

Choroid · Eyelid · Photodynamic therapy · Retinopathy · Risk factors

\begin{abstract}
We report a case of central serous chorioretinopathy (CSC) that developed 1 month after an intralesional injection of triamcinolone acetonide that was administered during removal of a chalazion. The subretinal fluid and ipsilateral visual acuity (VA) worsened with initial observation. The edema resolved with verteporfin photodynamic therapy (PDT) 1 month after diagnosis, but VA did not improve during short-term follow-up. We conclude that CSC can occur as a complication of low-dose intrapalpebral corticosteroid administration and provide another example of the therapeutic role of PDT in the management of this disease.
\end{abstract}




\section{Introduction}

Central serous chorioretinopathy (CSC) is characterized by the subretinal accumulation of serous fluid leading to localized neurosensory retinal detachment. Although the pathophysiology of this disease is not entirely understood, two main hypotheses have been supported. The disease has been suggested to occur as a result of increased choroidal capillary permeability leading to damage of the retinal pigment epithelium (RPE), which in turn causes subretinal serous fluid accumulation [1]. An alternative theory suggests that RPE dysfunction is the primary etiology of the subretinal edema [2]. Multiple reports have identified corticosteroid use and hyperaldosteronism as a potential risk factor for the development of this disease [3]. Spontaneous resolution occurs in $60 \%$ of cases of CSC within 3 months [3]. Verteporfin photodynamic therapy (PDT), among others, has been reported as a valid therapeutic alternative in persistent cases [2, 4]. In this report, we describe a case of CSC following the intrapalpebral injection of triamcinolone acetonide within the excision margins from the removal of a chalazion.

\section{Case Report}

A 28-year-old male presented for evaluation of recurrent, central right-upper lid chalazion previously treated with incision and curettage (I\&C). Conservative management with erythromycin ointment and warm compresses failed, prompting repeat I\&C. Given the chronicity of chalazion, we also administered a $0.5-\mathrm{mL}$ injection of triamcinolone acetonide $(10$ $\mathrm{mg} / \mathrm{mL}$ ) within the chalazion excision margins in an intrapalpebral fashion. One month following the procedure, the patient returned with concern of a "purple spot" in the central vision of his right eye (OD). OD examination showed unchanged visual acuity (VA) of 20/20, normal intraocular pressure, and intact visual fields. Fundus examination revealed subretinal fluid that was confirmed on optical coherence tomography (OCT) (Fig. 1a), which also revealed a small pigment epithelial detachment (PED) in OD (Fig. 1b); intravenous fluorescein angiography revealed pooling into the PED in the early phases along with an expansile dot pattern of leakage in later phases (Fig. 1c and d, respectively). The patient denied use of steroid-containing medications since presentation. At 1-month follow-up, VA OD had decreased to 20/50, and confrontational visual fields showed an ipsilateral central scotoma. OCT showed persistence of subretinal fluid with PED. He was treated with PDT with verteporfin $3 \mathrm{mg} / \mathrm{m}^{2}$ and 25 $\mathrm{J} / \mathrm{cm}^{2}$ laser fluence. Twenty days following the procedure, VA OD was 20/100 with resolution of the central scotoma. OCT obtained at this time showed resolution of the subretinal fluid (Fig. 2). The patient was lost to follow-up after this visit, and his visual status is unknown.

\section{Discussion}

Previous reports have correlated the onset of CSC with various modes of steroid use, including both systemic and local applications [5]. This disease has also been reported as a complication of sub-Tenon's corticosteroid injection [6].

CSC onset has been documented as early as 1 week following topical ocular and periorbital high-dose corticosteroid administration [6]. Our patient developed CSC within 1 month of the injection. We were unable to identify any literature clarifying the expected time frame between corticosteroid administration and CSC diagnosis. It is unlikely our patient developed 
CSC from another cause besides the intrapalpebral steroid injection, as there were no other established risk factors present for the condition.

The patient's subretinal edema and VA worsened with observation. Soon after treatment with PDT, the subretinal fluid and PED had resolved, although VA worsened. Subfoveal PEDs in CSC, as in our patient, have been found to have a poor visual prognosis with an average VA of 20/50 in one study [7]. We expected continued visual improvement over time after PDT; however, the patient was lost to follow-up. Regarding timeline to treatment, PDT and other therapeutic strategies are often initiated after 3 months for one isolated episode of CSC, due to the chance of spontaneous recovery during this period [2]. It is possible that our patient's CSC resolved on its own rather than as a result of the PDT given the natural time course of CSC, although the worsening VA and a new central scotoma were the reasons for initiating treatment with PDT.

In conclusion, our study identifies a new association between intrapalpebral low-dose corticosteroid administration and development of CSC and reports complete resolution of subretinal fluid with early phototherapy. To our knowledge, this is the first report of CSC secondary to injection of triamcinolone within the excision margins following chalazion excision.

\section{Statement of Ethics}

Informed consent for this case report was not obtained prior to our patient being lost to follow-up. However, no identifying information is included in this case report. The study was carried out without approval from our Institutional Review Board, as none is needed from our institution for case reports.

\section{Disclosure Statement}

Our institution receives an unrestricted grant from the Foundation of Research to Prevent Blindness (RPB) to support research and publications. RPB has no conflicts of interest with this work. This research did not receive any specific grant from funding agencies in the public, commercial, or nonprofit sectors. The following authors have no financial disclosures: L. Rosignoli, S. Potter, A. Gonzalez, S. Amin, S. Khurshid.

\section{Author Contributions}

Luca Rosignoli: Participated in drafting the manuscript, collection, analysis and interpretation of the data. Read and approved the final manuscript. Stephen Myles Potter: Participated in drafting the manuscript, collection, analysis and interpretation of the data. Read and approved the final manuscript. Andres Gonzalez: Participated in drafting the manuscript, collection, analysis, and interpretation of the data. Read and approved the final manuscript. Sarina Amin: Participated in drafting the manuscript, collection, analysis, and interpretation of the data. Read and approved the final manuscript. Syed G. Khurshid: concepted, analyzed, interpreted the data and revised it critically for intellectual content. Read the final manuscript and gave final approval of the version for submission. 


\section{References}

1 Ross A, Ross AH, Mohamed Q. Review and update of central serous chorioretinopathy. Curr Opin Ophthalmol. 2011 May;22(3):166-73.

2 Gemenetzi M, De Salvo G, Lotery AJ. Central serous chorioretinopathy: an update on pathogenesis and treatment. Eye (Lond). 2010 Dec;24(12):1743-56.

3 Gong Q, Sun XH, Yuan ST, Liu QH. The relation of the serum aldosterone level and central serous chorioretinopathy - a pilot study. Eur Rev Med Pharmacol Sci. 2017 Feb;21(3):446-53.

4 Wang M, Munch IC, Hasler PW, Prünte C, Larsen M. Central serous chorioretinopathy. Acta Ophthalmol. 2008 Mar;86(2):126-45.

5 Balakrishnan S, Apsingi S, Manjure SB. Sudden loss of visual acuity following intra-articular steroid injection in to the knee joint: a case report. Cases J. 2008 Dec;1(1):428.

6 Baumal CR, Martidis A, Truong SN. Central serous chorioretinopathy associated with periocular corticosteroid injection treatment for HLA-B27-associated iritis. Arch Ophthalmol. 2004 Jun;122(6):926-8.

7 Mudvari SS, Goff MJ, Fu AD, McDonald HR, Johnson RN, Ai E, et al. The natural history of pigment epithelial detachment associated with central serous chorioretinopathy. Retina. 2007 Nov-Dec;27(9):1168-73.
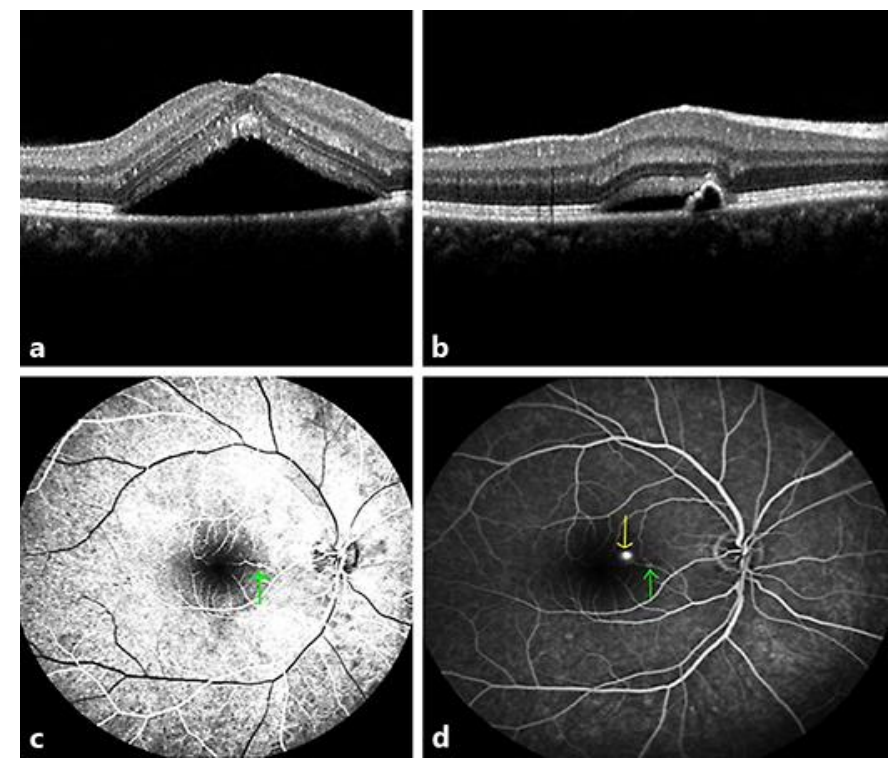

Fig. 1. Spectral domain OCT showing preprocedure subretinal fluid (a) with PED (b). Standard intravenous fluorescein angiography in the early arteriovenous phase at $16 \mathrm{~s}$ (c) showing pooling into the PED (green arrow) and in the venous phase at $31 \mathrm{~s} \mathrm{(d)} \mathrm{showing} \mathrm{an} \mathrm{expansile} \mathrm{dot} \mathrm{pattern} \mathrm{(yellow} \mathrm{arrow)} \mathrm{with}$ pooling into the PED (green arrow). 


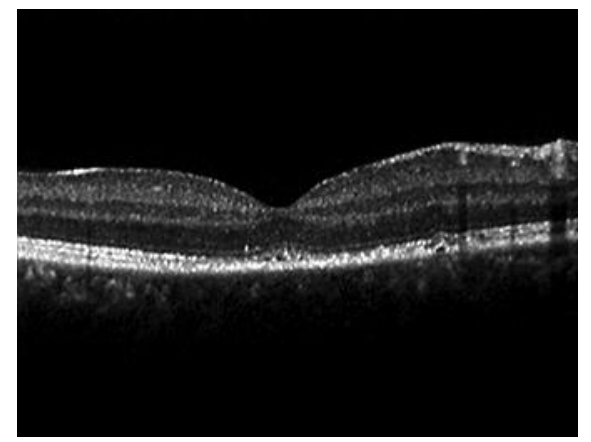

(c) 2018 The Author(s). Published by S. Karger AG, Basel www.karger.com/cop

Fig. 2. Spectral domain OCT after PDT showing resolution of SRF. 